Cinémas

Revue d'études cinématographiques

Journal of Film Studies

\title{
La Berlue et le mythe : $S / K$, ou de Stephen King à Stanley Kubrick
}

\section{Jean-Pierre Vidal}

Volume 4, numéro 1, automne 1993

Écrit/Écran

URI : https://id.erudit.org/iderudit/1000115ar

DOI : https://doi.org/10.7202/1000115ar

Aller au sommaire du numéro

Éditeur(s)

Cinémas

ISSN

1181-6945 (imprimé)

1705-6500 (numérique)

Découvrir la revue

Citer cet article

Vidal, J.-P. (1993). La Berlue et le mythe : S/K, ou de Stephen King à Stanley Kubrick. Cinémas, 4(1), 115-129. https://doi.org/10.7202/1000115ar
Résumé de l'article

L'auteur analyse ici Shining du point de vue de l'adaptation que fait Kubrick du roman de Stephen King. Il montre que la mise en images du roman représente un travail de surexposition et de distanciation de la lecture comme telle, donnant une réalité " objective " à des images sans contour que produit toute lecture, opérant dans le dévoilement de sa propre lecture une représentation concomitante du regard piégé du spectateur. Le travail sur le texte laisse ainsi apparaître comme piste de lecture un récit sans texte, une sorte de fantasme, le mythe peut-être de toute création artistique. 


\title{
La Berlue et le mythe : S/K, ou de Stephen King à Stanley Kubrick
}

\author{
Jean-Pierre Vidal
}

\begin{abstract}
RÉSUMÉ
L'auteur analyse ici Shining du point de vue de l'adaptation que fait Kubrick du roman de Stephen King. Il montre que la mise en images du roman représente un travail de surexposition et de distanciation de la lecture comme telle, donnant une réalité «objective» à des images sans contour que produit toute lecture, opérant dans le dévoilement de sa propre lecture une représentation concomitante du regard piégé du spectateur. Le travail sur le texte laisse ainsi apparaître comme piste de lecture un récit sans texte, une sorte de fantasme, le mythe peut-être de toute création artistique.
\end{abstract}

\begin{abstract}
The author analyses Kubrick's The Shining, adapted from the novel by Stephen King. He shows that the filming of the novel represents a work of overexposing and distancing from reading as such, giving an "objective" reality to the formless images derived from any reading, producing through the revelation of its own reading a concomitant representation of the entrapped gaze of the spectator. The work on the text thus opens the way for the appearance as a reading path of a story without text, a sort of fantasy, perhaps the myth of all artistic creation.
\end{abstract}


Dans la mise en images qu'il fait du roman de Stephen King dont, significativement, il garde le titre - à l'article près : Shining -, Kubrick rejoint la pratique d'un autre grand cinéaste «s'inspirant» tout autant, malgré les apparences, mais d'une toute autre façon, d'un texte dont, non moins significativement, il ne garde pas le titre : Francis Ford Coppola tournant Apocalypse Now dans les marges de Heart of Darkness de Joseph Conrad. Les deux films sont contemporains (1979 pour Apocalypse Now et 1980 pour Shining ), ce qui peut expliquer la parenté que mon regard leur attribue et surtout la configuration intertextuelle particulière que ce même regard dessine entre eux : les textes que ces films transforment en images ont un horizon commun, sous la forme d'une référence à mon sens éclatante, au mythe de Dédale. Qu'il y ait à ce moment, chez deux cinéastes reconnus pour leurs tendances baroques, un même appel de ce mythe particulier tient sans doute au rapport entre la métaphore de la création artistique (c'est cela sans doute, vu sous l'angle de Dédale, le labyrinthe) et la guerre qui pousse les pères à liquider les fils (Dédale est bien la figure de ce père-là et l'aventure de Thésée est, comme celle d'Edipe dont elle est le pendant, l'aventure d'un parricide qui mène au pouvoir). C'est sans doute sous l'angle du motif de la maîtrise comme liquidation de l'immaturité que le mythe de Dédale réunit la guerre, ou plus généralement la violence (et de préférence intime), et l'art. Une telle conjonction n'est sans doute guère politically correct et gardera toujours quelque chose de profondément choquant pour les âmes sensibles que nous sommes, mais l'artiste assassin (des Beaux-Arts considérés comme un assassinat?) n'est pas une invention du $\mathrm{XX}^{\mathrm{e}}$ siècle et les effets d'une telle conjonction entre l'art et le meurtre ne doivent pas se lire dans un contexte axiologique trop pragmatique. Dans les deux films, les personnages principaux sont expressément présentés comme des artistes «et» des tueurs, et même plus : des tueurs "parce» que des artistes : Torrance, dans Shining, est un écrivain qui se fait gardien des lieux pour pouvoir travailler; quant à Kurtz, dans Apocalypse Now, son grand dessein, aussi mystérieux et non explicité chez Coppola que chez Conrad, est manifestement une œuvre, fût-elle de mort, et le photographe qui lui sert de fou du roi le dit bien, en forme de présentation, aux passagers de la 
vedette : "Man, he's a poet. He has enlarged my mind", ce qui est une citation exacte du texte de Conrad placé dans la bouche d'un personnage équivalent.

Comment le mythe, donc, au-delà des textes où sa présence se marque aussi, à un niveau plus thématique chez King, plus narratif chez Conrad, se lit-il dans les images et le discours du film? En quoi sa convocation discrète dans les films représente-telle l'infléchissement que ceux-ci font subir aux textes, dont il représente dès lors la lecture en forme de leçon?

Étant donné la difficulté d'envisager les deux films dans le cadre de cet article, je me limiterai à Shining, à propos duquel je m'efforcerai de montrer que sa mise en images du roman de Stephen King représente un travail de surexposition et de distanciation de la lecture comme telle, travail qui dans le gauchissement qu'il opère des formes narratives du texte en transforme les «images». Le cinéaste, en donnant une réalité «objective» à ces images sans contour que produit toute lecture, opère dans le dévoilement de sa propre lecture une représentation concomitante du regard piégé du spectateur.

Ce travail, visible et visuel, sur un texte fait ainsi apparaître comme piste de lecture un récit sans texte, une manière de fantasme donc, un mythe, «le» mythe peut-être de toute création artistique, mise en abîme majuscule et absolue : celui, je l'ai dit, de Dédale et de son labyrinthe. Comme si l'espace du film produisait cette référence au moment même où il fonde sa spécificité sur la recomposition/décomposition d'un texte. C'est sans doute que la mise en image d'un récit déjà fait en produit une variante qui aussitôt (c'est la logique du mythe, «ensemble de ses variantes» dit Lévi-Strauss) s'y résorbe en en étendant l'aire signifiante. Cette aire élargie, jusqu'à conjoindre non seulement diverses articulations narratives mais aussi divers matériaux d'énonciation, prend l'aspect d'une anamorphose en donnant à la dimension iconique des référents du texte des contours que, par définition, la lecture ne peut préciser. Ce travail est particulièrement évident, comme je l'ai déjà dit, chez Kubrick mettant en images le texte de King comme s'il plaçait sur ces référents iconiques sans contours un calque qui leur donne une forme reconnaissable, non seulement, comme il est inévitable au cinéma, en termes d'effets de réel, de prise de corps en quelque sorte, mais aussi et surtout, dans la mesure où l'artifice cinématographique ici, au lieu de se cacher, se met en évidence ironiquement, en termes de lecture orientée vers une amplification du récit, déjà second, jusqu'à cette matrice de la pléthore des récits qu'est le mythe. 
Voyons comment s'opère ce travail à partir de quelques exemples choisis pour leur rapport direct à la trame du récit, et à celle du mythe en même temps. Pris dans sa globalité diégétique, The Shining de Stephen King est l'histoire d'une projection : celle d'un espace, le palace, et des divers temps qu'il renferme (de l'«année dernière» au début du siècle à quoi remonte sa construction) sur le temps de la famille du gardien, projection à laquelle s'oppose la contre-projection du don de voyance (shining) de l'enfant et du cuisinier noir, contre-projection dont l'effet de vérité se dit en forme de spécularité par le palindrome redrum où se lit le meurtre ${ }^{1}$ (murder), comme à la fois passé et futur (fonction que remplit généralement le hors-champ au cinéma, si l'on en croit Aumont ${ }^{2}$ ), permanence de l'espace, ainsi proprement labyrinthique ${ }^{3}$, ce que le film met fort habilement en images sous la forme d'une inscription sanglante sur une porte de chambre $^{4}$ : le palace exsude le meurtre, comme si ses portes, carrefours du labyrinthe, étaient aussi des pores. Quant au temps familial, tout au plus peut-on dire que le palace, dans la pluralité d'époques et de crimes qu'il a connus, voit peu à peu émerger l'autre temps susceptible de capturer celui de la famille Torrance, par analogie, temps familial lui aussi, celui de la famille Grady, le précédent gardien, mais aussi celui de la famille qui peuple le labyrinthe de Dédale. La synecdoque majuscule du palace produit une métaphore médusante qui se résorbe en synecdoque quand la famille Torrance est capturée par le palace et ses ombres. Dans le roman de King, le destin ordinaire est capturé progressivement par les destins d'exception qui hantent les chambres du palace. Histoire de maisons hantées et de vampires une fois de plus. Mais qui, comme toujours chez King, rencontrent une culpabilité individuelle (Torrance est un alcoolique doublé d'un bourreau d'enfants) qui s'y trouve une justification masochiste.

Shining de Kubrick est aussi l'histoire d'une projection, mais d'entrée de jeu, sans passer par la multiplicité des vies du palace, celle d'une vie sur une autre, d'un espace privé sur un autre, le cadre du palace valant moins comme espace public contextuel que comme cadre «naturel», ce que montrent fort bien l'ouverture par la monumentalité du décor montagnard et, plus tard, le labyrinthe végétal. Il y a, en effet, véritablement une stratégie des lieux à l'œuvre dans ce film, des montagnes à la chambre froide et du labyrinthe végétal à la chambre secrète, stratégie des lieux qui fait écho à celle que l'on trouve dans le texte, notamment à propos du travail de maturation de l'écriture chez Torrance 5 . Une vie, donc, se projette sur une autre, le 
précédent gardien se redouble sur le gardien actuel en une étreinte spéculaire et agonistique, et les deux se résorbent ensemble dans cette énigmatique image finale où Nicholson, qui joue, on s'en souvient, le rôle du gardien actuel, apparaît dans la photo d'un bal des années 20. Ce bal dans le palace, c'est le retour du multiple qui fait ainsi pendant au jour de la fermeture annuelle par quoi, après les séquences de montagne, s'ouvre, avec «l'entrevue», le film. Kubrick, on le voit, introduit le motif labyrinthique, non point tant au niveau du décor, encore que celui-ci soit explicitement travaillé dans ce sens, qu'à celui de la spécularité de l'image, Narcisse-Nicholson capturé par un reflet qui n'est pas le sien, représentation on ne peut plus frappante du processus de projection et d'identification, maintes fois analysé, qui saisit le spectateur de cinéma. N'oublions pas que si le plan précédant immédiatement la photo finale vient de montrer Torrance gelé au cœur du labyrinthe végétal où son fils a réussi à le perdre (vient donc de le montrer incorporé au paysage et au temps météorologique comme ici il s'incorpore au palace et au temps de ses commencements), une séquence entière, à peu près au centre du film, dans laquelle Torrance a rencontré Grady et s'est fait en quelque sorte passer le relais par lui (séquence traitée sans la moindre marque d'hallucination ou de fausseté), a rendu présentes les fêtes d'autrefois, comme une énigme du passé et un piège du futur, un hors-champ venu dans le champ à la rencontre du personnage dont la caméra dès lors matérialise le regard, comme une projection de l'intérieur de sa tête. Si les séquences ne sont pas traitées en "caméra subjective», c'est précisément pour montrer ce chevauchement des temps et des espaces, ce collage rendu évident par la présence dans le champ du personnage qui voit, collage et chevauchements qui ont l'effet d'un montage résorbé, d'une surimpression où, contrairement à ce qui se passe avec le procédé de la «caméra subjective», qui connoterait plutôt dans un tel cas l'hallucination et donc la distance au spectateur, c'est le regard du spectateur lui-même qui est pris et peut-être ironisé dans ce qui s'énonce comme un trompe-l'œil manifeste.

Dans la photo finale se disent aussi, à mon sens, l'immobilisation du temps et du film dans le pseudophotogramme 6 , la saisie de l'image par le spectateur et la capture, par elle, de ce même spectateur que le dispositif optique somme d'assumer son individualité au sein du public (j'y reviendrai). Le Barton Fink de Joël Coen, que je considère personnellement comme un chef-d'œuvre, et qui pourrait bien être, de façon assez subtile, une reprise du film de Kubrick 
(écrivain, hôtel, meurtre, photo sur mur), met en scène un procédé analogue mais dont les différences permettent de mieux éclairer ce qui nous occupe ici : ce n'est plus sur un pseudophotogramme que se termine le film, mais sur un plan fixe, l'un comme l'autre matérialisant diversement le regard du spectateur, invité dans un cas (Kubrick) à prendre congé du personnage qui le regarde en forme de point final, dans l'autre au contraire à suivre le personnage au bout de son propre regard, dans la mesure où Fink est alors rentré dans l'image qu'il contemplait sans cesse dans sa chambre (cette jeune femme au bord de la mer), mais cette image est maintenant animée, non pas comme il est courant (par exemple, Nous nous sommes tant aimés de Scola) par l'aboutissement d'un travelling avant (où «l'œil» de la caméra a l'effet d'une mémoire et, se rapprochant, ranime ce qui était figé, revisualisant ainsi à chaque fois en quelque sorte le mythe fondateur du cinéma, de l'image à sa multiplication en forme de simulacre de mouvement), mais comme une immobilisation en plan fixe de cet œil seul, et non de l'image qui, elle, reste animée, comme si la "réalité» ainsi toujours visiblement échappait, dans son flux, au cadre figeant.

Dans Shining, cette image finale s'oppose manifestement à la fluidité des immenses travellings d'ouverture qui, en succession, montrent du haut des airs la progression de la voiture de Torrance dans un paysage montagneux. Elle s'oppose aussi à la séquence immédiatement antérieure, la poursuite en forme de travellings avant rapides dans le labyrinthe végétal, comme si le film, annoncé comme tel spectaculairement dans la séquence d'ouverture et rejoué en coda mouvementée dans la séquence du labyrinthe, se résorbait en photogramme ${ }^{7}$ et plus encore en ce regard que Nicholson renvoie, de sa photo, à celui du spectateur. En ce sens, Shining fait coïncider la fin de sa fiction avec celle du spectacle, reflet bientôt immobile dans un œil qui se referme sur lui. Et cette fin est une agression, ou tout au moins une menace, le spectateur ne pouvant sans doute s'empêcher de penser qu'il est le prochain sur la liste du vampire et que c'est le cinéma tout entier qui, telle Méduse, pétrifie sa vie en forme de simulacre et tantôt peut-être le capturera.

Car Shining est bien le film de la contagion par l'imaginaire, le film de la "projection», celle qui va de la salle à l'écran et que matérialisent fort bien les travellings du début mais surtout celle, inverse, perverse et pour tout dire diabolique - ou «fatale» au sens des «stratégies» de Baudrillard — qui va de l'écran à la salle et traduit remarquablement dans un autre langage le credo implicite de Stephen King8 que la monstruosité est l'effet de réel 
de l'imaginaire, ce qu'illustre fort bien It. Encore faut-il ajouter aussitôt qu'un tel effet provient ici du véritable face à face que produit, entre le spectateur et l'image fixe de l'acteur, le très lent travelling vers la photo, puis le montage qui en rétrécit le champ et par lequel Kubrick agrandit le hors-champ aux dimensions de l'univers, laissant le regard du spectateur cloué à une spécularité agonistique. Nicholson, que nous avons appris à identifier au personnage de Torrance, se trouve épinglé dans la photographie, à la place du premier gardien de l'hôtel qui seul peut diégétiquement avoir vécu le bal dont la photo porte témoignage. Dans ce jeu de substitution, c'est Torrance qui visiblement se trouve effacé, alors que l'acteur, véritable joker, d'être ainsi à la place de celui qui remplace, redevient paradoxalement Nicholson, à mesure que le cadrage évacue dans le hors-champ tout ce qui n'est pas son visage, Nicholson... ou n'importe qui, et aussi bien moi que le piège optique, dès lors attend. Autrement dit, le visage célèbre de Nicholson s'ouvre sur l'inconnu anonyme que l'image attend et le film a vampirisé son spectateur.

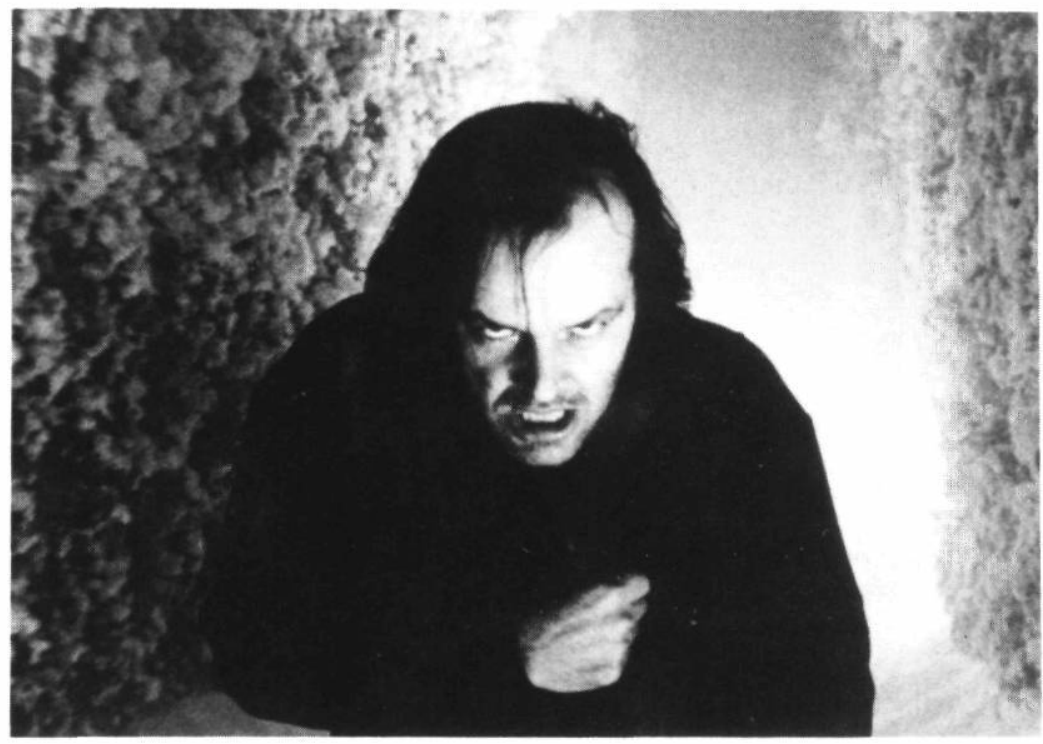

Shining de Stanley Kubrick

Coll. Cinémathèque québécoise 
Ce type d'effet, bien entendu, est impossible dans un texte, mais on concédera cependant que la lecture, avec la projection qu'elle induit sur le lecteur, projection dont le texte à la fois se constitue et joue, en est l'équivalent. Ainsi peut-être Kubrick met-il littéralement en scène sa lecture du roman, renvoyant vers le spectateur, par le travail effectué sur le texte (travail qui permet de s'en déprendre sans doute puisqu'il est alors question d'en discerner les articulations, d'en poursuivre et d'en modifier le jeu par visualisation), l'effet médusant de capture. Et la lecture que je fais ici du film, loin de me renvoyer à la littérature de Stephen King, m'en éloigne, à la fois en la démultipliant (la «transcription» qu'est le film et la transcription seconde que ma lecture en fait) et en la réduisant (la modification de l'architecture du livre ramenée d'une part à sa diégèse ou, si l'on préfère, au plan du contenu, et d'autre part simplifiée pour faire apparaître, dans son foisonnement événementiel et littéral, quelques symétries, quelques rapports, jusque-là insoupçonnés et peut-être même insoupçonnables, symétries et rapports sur lesquels le film assoiera sa propre architecture, mais aussi symétries et rapports que ma lecture seule fait apparaître sans doute).

Cet infléchissement du texte, que la lecture produite par le film ordonne dans le sens du mythe, va se marquer aussi, outre la représentation de son mode de consommation spécifique ${ }^{9}$ telle que j'ai ici tenté de la mettre en évidence, par une double transcription, en forme de déplacement pour l'une, de condensation pour l'autre : la double hache de Thésée est représentée dans le livre (mais peut-être ne l'y voit-on qu'après avoir vu le film) par un maillet d'un jeu — le «roque» - que le texte présente comme l'ancêtre du croquet, dans le passage cidessous où l'on ne manquera pas de voir un effet labyrinthique dans la comparaison de ce jeu d'extérieur à un jeu d'intérieur. C'est Torrance qui parle à son fils : "It's a little bit like croquet, only you play it on a gravel court that has sides like a big billiard table instead of grass. It's a very old game, Danny. Sometimes they have tournaments here" (p. 68). La lecture peut sans doute ne pas percevoir la menace dans les tournois de roque annoncés ici par le père à son fils, mais la suite immédiate ne la lui laissera pas ignorer: "Do you play it with a croquet mallet" demande Danny, et Torrance de répondre : "Like that (...) Only the handle is a little shorter and the head has two sides. One side is hard rubber and the other side is wood". Aussitôt après apparaît, entre parenthèses et en italiques, une phrase de shining : "Come out, you little shit!"(p. 68). Elle renvoie aux nombreuses autres 
qui tout au long du texte, et depuis le début, représentent une voix avinée - bien sûr celle, proleptique, de Torrance - qui résonne par shining dans la tête de l'enfant. Ici, de façon significative, elle est suivie immédiatement par "It's pronounced roke, Daddy was saying, I'll teach you how to play, if you want" (p. 68). La double entente de ce «je t'apprendrai à jouer» est particulièrement évidente dans un tel contexte. Kubrick réduit cet échafaudage complexe. Le film qui, incidemment, ne connaît pas de prolepse, comme il serait possible, dans la mesure où toutes les scènes de violence à venir sont déjà passées, met entre les mains de Torrance non pas un maillet, comme dans le livre, mais une hache d'incendie qui provient du meurtre familial antérieur (ce que dit aussi le texte : Grady a tué ses filles avec une hachette, sa femme puis lui-même avec un fusil). Ce déplacement, bien entendu, rend plus évidente la présence du mythe dont il inscrit un des éléments majeurs qui dans le texte demeurait plus secret (la double tête du maillet) et plus diffus, convoqué qu'il était par un véritable procédé de montage : jeu en forme de labyrinthe, tournoi, le meurtrier menaçant sa victime de lui apprendre à jouer, après qu'une phrase venue de nulle part ait contextualisé différemment cette offre.

Quant à la condensation que j'annonçais précédemment, la voici, à partir de la même page du texte à laquelle je reviens. Torrance vient de dire à son fils qu'il ne sera pas obligé (!) de jouer au roque s'il n'en a pas envie et c'est aussitôt Wendy, sa femme, qui parle : "Do you like the animals? (...) That's called a topiary". Ce qui est ainsi annoncé à l'attention de l'enfant c'est ce que le texte décrit de la façon suivante : Beyond the path leading to roque there were hedges clipped into the shapes of various animals. Danny, whose eyes were sharp, made out a rabbit, a dog, a horse, a cow and a trio of bigger ones that looked like frolicking lions". La topiaire et le roque, termes aussi rares en anglais qu'en français, connotent ici la menace adressée à l'enfant, par leur rareté même, car celle-ci matérialise l'insuffisance de sa compétence linguistique, mais aussi parce que s'agissant de la topiaire, Torrance en fait la raison pour laquelle on l'a engagé : il a déjà taillé des haies, autrefois, pour une femme, dont aussitôt Danny demande : "Did she have nice hedges?", ce qui fait éclater de rire son père et sa mère, le double sens un peu grivois cette fois produit innocemment par l'enfant faisant écho au double sens menaçant produit tout à l'heure par le père. Ici encore, Kubrick simplifie : il écrase l'un dans l'autre la topiaire et le roque, les condensant en ce labyrinthe végétal, double de l'hôtel, et où tout va se jouer, le 
père y poursuivant son fils avec la hache en guise de maillet. Dans le roman, ce sont les animaux de la topiaire qui s'animeront, ce qui ne peut inscrire le mythe que pour qui connaît l'animation des statues attribuée aussi à Dédale, à moins que la combinatoire du mythe ne soit, comme le postule d'ailleurs Lévi-Strauss, une structure logique induisant de façon récurrente les mêmes faisceaux de métaphores d'elle-même, l'animation des objets allant ici de pair avec l'immobilisation de l'espace. Mais dans le roman, cette animation de la haie continuera de pluraliser les forces maléfiques 10 qui s'attaquent à la famille. Dans le film, au contraire, ces haies animales se referment en lieu clos où l'espace, comme le temps, se répercute.

Mais la simplification dont je fais état va plutôt dans le sens d'une raréfaction de certains éléments vecteurs du récit, que le film dans son déroulement met en évidence, alors que le texte les dissémine dans son foisonnement : comme si le film, aux prises avec le réel innombrable et donc ainsi menacé de perdre son fil narratif dans la pléthore qui s'inscrit visiblement dans tout plan, devait raréfier le texte et ses quasi incernables effets visuels pour y puiser une armature. C'est ainsi, par exemple, que le film de Kubrick va inscrire dans le motif labyrinthique quelque chose qui, dans le roman, ne joue qu'un rôle accessoire et diffus, réductible à la limite à un effet de réel : le véhicule.

Dans Shining, en effet, le moyen de transport sert à répercuter l'effet labyrinthique, proprement cinématographique, qu'est le passage, disons, du très gros plan au plan général, ou l'inverse, d'un microcosme à un macrocosme, ou plus exactement d'une microscopie à une macroscopie, ou l'inverse : la voiture jouet du jeune garçon fonçant dans les couloirs de l'hôtel miniaturise la séquence d'ouverture qui voit monter les Torrance, dans leur «coccinelle» vers l'hôtel11. Elle sera aussi l'un des stades intermédiaires du signifiant «véhicule» dont la chenillette grâce à laquelle l'enfant et sa mère pourront quitter l'hôtel à la fin sera le stade ultime, dédoublé, dans la mesure où c'est non pas la chenillette de l'hôtel, immobilisée par le sabotage de Torrance, mais celle du cuisinier assassiné qui permettra leur fuite. Auparavant, la voiture de l'enfant elle-même se sera dédoublée, le "chapitre VI», "Mercredi» s'ouvrant sur un plan de Danny jouant avec des petites voitures sur le tapis au motif labyrinthique qu'il parcourait auparavant dans sa voiture à pédales. Comme pour mieux établir le rapport, une balle roule vers lui, l'attirant, à pied cette fois, hors de son jeu, vers ce qu'il suppose être son origine, au bout de l'interminable couloir. Elle servira aussi, avec la machine à écrire et la balle de baseball 
lancée contre un mur par Torrance, à composer, dans l'alternance du bruit sourd de son roulement sur les tapis, sonore sur la pierre, la répercussion répétitive par laquelle la bande-son dit elle aussi, comme nous l'avons vu, le motif labyrinthique. Elle servira enfin, par la miniaturisation accompagnée de la transcription du parcours de l'extérieur à l'intérieur qu'elle représente par rapport à la séquence d'ouverture, et par la miniaturisation qu'elle-même à son tour subit quand l'enfant, comme on vient de le voir, joue sur une miniaturisation du labyrinthe du tapis, non seulement à lier, comme je l'ai déjà dit, cette séquence d'ouverture à la séquence finale, mais aussi à «résoudre», comme on parle de résolution en musique, toutes ces miniaturisations successives, la séquence de l'enfant jouant avec ses petites voitures succédant à cette séquence extraordinaire qui, dans le «chapitre III», «Un mois plus tard» a montré Torrance regardant la maquette du labyrinthe végétal et, devenu ogre gigantesque par ce regard même, y «voyant» jouer (en fait, c'est le regard de la caméra qui relaie son regard et le nôtre, les conjoignant ainsi visiblement), de vertigineusement haut, son fils et sa femme. Le film de Kubrick organise ainsi résolument un labyrinthe plus optique qu'uniquement spatial : c'est le film tout entier qui est un labyrinthe, non pas à la manière de L'Année dernière à Marienbad, par le montage, la recomposition incessante des plans 12 et l'ambiguïté du jeu avec le décor, mais par cette menace constante d'un agrandissement ou d'un rétrécissement, également vertigineux, du champ, agrandissement ou rétrécissement auxquels participe aussi la bande-son qui inscrit ainsi une autre des vérités du mythe : qu'à la limite, le labyrinthe est plus une machine et même un rythme, quand ce n'est pas une aporie, qu'un édifice ${ }^{13}$, fluctuations «à vue» du champ qui ont pour corollaire l'institution de la salle où se tient le spectateur en hors-champ «provisoire» et donc la menace qui pèse sans cesse sur ce même spectateur que, se retournant, il puisse se voir non pas dans le regard rassurant du spectateur d'en arrière mais dans l'œil impassible et le champ supposé d'une monstrueuse caméra. Encore l'effet vampirique dont je parlais tout à l'heure. Le syndrome des Carabiniers ou de The Purple Rose Of Cairo.

Une mise au point, pour finir, sur la façon dont j'ai lu le mythe en filigrane dans la lecture que le film fait du livre. On aura remarqué peut-être que je me soucie peu d'énoncer des correspondances parfaites entre les éléments de l'un et l'autre discours, de voir dans Torrance-Nicholson, par exemple, une représentation de Thésée, ou de Dédale ou du Minotaure, sa 
femme Wendy restant Ariane, son fils Danny ne pouvant être que Thésée ou Icare ${ }^{14}$. Mais c'est d'une part que Torrance occupe manifestement les trois fonctions, représente les trois figures : il devient Minotaure ${ }^{15}$ dans la mesure où il échoue à être Thésée face au Dédale que représente Grady, le premier gardien. Il assume, en relais de ce dernier, la position du père meurtrier dont, devenu une bête, il est à la fois le remords et l'action. C'est surtout, indépendamment du cas particulier que représente le film, que le mythe, comme le rêve, repose sur un dynamisme métamorphique qui court à la surface du récit, jouant de la métonymie et plus encore de l'hypallage pour construire un code à dire des énigmes plus qu'à les expliciter.

Le mythe est un discours dont non seulement les articulations narratives mais l'existence même en tant qu'elle se distingue des institutions de la tribu n'est perceptible que dans l'analyse ou la lecture qu'on en fait. Sans le regard porté sur lui, s'en déprenant, le mythe n'est qu'une cérémonie ou un récit répercuté. Mais sa lecture est, du même coup, une de ses variantes. Peut-être n'échappe-t-on jamais au mythe pour cette raison que le lisant on $y$ ajoute, on s'y ajoute, avec les histoires non abouties que l'on a dans la tête, et ainsi on s'y perd.

Quoi qu'il en soit, et n'en déplaise à la mythocritique, ce n'est pas par son contenu, d'ailleurs toujours problématique, que le mythe informe l'œuvre d'art. C'est plutôt par le jeu des formes qu'il déclenche et le labyrinthe signifiant que toujours, quel que soit son propos et si tant est qu'on puisse le discerner, il ordonne. Comme tel, il offre une analogie maintes fois signalée avec l'œuvre et le mythe de Dédale, mythe de la maîtrise de l'espace et du temps, mythe des âges de la vie et de la maturation, mythe de l'accomplissement comme perte du sujet dans le lieu et les formes de son action, mythe de l'art, et il pourrait bien être aussi le mythe du mythe. En ce sens que, contrairement au discours de la science, et semblablement à celui de l'œuvre, son discours reste ouvert, sa forme tout au plus une partition, son labyrinthe tout au plus une piste hasardée de laquelle un architecte fou, moi, vous, érige, l'immobilisant pour un temps, pour «son» temps, des murs comme autant de cénotaphes.

Et si tout ça n'était, jusqu'à la berlue, que le cinéma qu'on se fait? À partir, toujours, du texte des autres, des textes de l'Autre...

Université du Québec à Chicoutimi 
1 Ce qui se lit peut-être d'abord, en fait, dans ce signifiant en forme d'apax, c'est la dimension acoustique, ce son rocailleux, cette répétition martelée des $r$ qui peut bien dès lors se résorber et se réduire à un signifiant identifiable, comme un commentaire et une désignation : red drum, tambour rouge. D'ailleurs, ce commentaire implicite sera explicité un peu plus loin, lorsque l'enfant, intrigué par ce mot qui résonne dans sa tête, demandera à son père : "What's redrum" et que ce dernier répondra : "Red drum? Sounds like something an Indian might take on the warpath" ( p. 128). Plus loin encore, un médecin consulté proposera plutôt que drum, "rum. As in the drink, the alcoholic drink", ce qui provoquera chez Torrance qui se sent ainsi accusé la réaction suivante : "Oh!, Jack said. It fits in, doesn't it?" (p. 151). Il me semble que le film de Kubrick, par l'entremise du bruit fait sur les surfaces diverses du plancher par la voiture à pédales de l'enfant et de ceux, liés, de la machine à écrire et de la balle de tennis lancée de façon répétitive sur un mur par Torrance-Nicholson, tiendra compte davantage de la leçon «tambour». On voit bien, quoi qu'il en soit, que le livre fait, quant à lui, de ce signifiant à géométrie variable, un motif labyrinthique.

2 Voir L'Eil interminable (Paris : Séguier, 1989).

3 Cette permanence, le film l'assure encore de faisant que la fin ne soit «que» la mort de Torrance et celle du cuisinier noir, alors que le roman (où le cuisinier noir réussit dans sa tentative de sauver femme et enfant, devenant une sorte de père substitut, shining obligeant) fait exploser l'hôtel, parce que Torrance a négligé de surveiller la chaudière. Le temps est présenté comme rythme abolissant l'espace qui n'a pu contenir son accélération, sa dilatation, le crescendo de sa récurrence.

4 À noter que cette inscription, c'est l'enfant qui la fait, vers la fin du film, juste avant l'apparition fracassante de Torrance avec sa hache. Jusque-là Redrum n'a été qu'un mot dans sa bouche, un mot prononcé par la voix de son double de l'autre côté du shining et du miroir: Tony. Le roman, quant à lui, assignera à cette inscription une autre stratégie : outre le jeu typographique qui l'inscrit en standard majuscule alors que ce qui, comme ce mot, représente l'émergence du shining dans la tête de l'enfant se marque en italiques, le terme s'inscrit dans un rapport de couleurs, de matières et de lieux d'apparition qui manifestent une véritable réécriture. Qu'on en juge plutôt en comparant la première apparition du terme ( New York : Signet books, 1978, p. 32 : "Across the room was a mirror, and deep down in its silver bubble a single word appeared in green fire and that word was : REDRUM") à celle de la fin de la première partie ("At last he crept back into his bed (...) and watched the shadows thrown by the alien streetlight turn into a sinuous jungle filled with flesh-eating plants that wanted only to slip around him, squeeze the life out of him, and drag him down into a blackness where one sinister word flashed in red : REDRUM", p. 57). On voit que le feu vert de l'inscription est devenu l'univers végétal qui mène à l'obscurité où cette fois s'inscrit le mot, serti précédemment au contraire dans une «bulle argentée», la projection des lumières de la rue à l'intérieur de la chambre représentant l'inverse du regard de tout à l'heure plongé au plus profond du miroir. La différence tient aussi à l'apparition de qualificatifs focalisateurs : alien streetlight, sinister word.

5 Dans le roman, c'est une pièce de théâtre qu'il cherche à écrire après avoir publié quelques nouvelles et tout en sentant un roman qui s'agite en lui : "(...) there might be a novel incubating in some mental backroom" (p. 38). La métaphore de la chambre mentale produit au moins deux effets. D'une part elle fait de la tête de Torrance l'équivalent miniaturisé de l'hôtel et ainsi, ce qui 
«incube» ici répond à ce qui (la chaudière) là voit sa pression monter au point, à la fin, de faire exploser tout l'hôtel, Torrance, dont c'est une des tâches, ayant oublié de la surveiller. La tête de Torrance elle aussi explosera littéralement. Cette métaphore de la chambre mentale produit aussi, bien sûr, une mise en abîme en forme d'invagination : le roman qui incube, c'est celui de la chambre secrète qui, dans la fiction, attend Torrance, c'est-à-dire que le roman, une fois résorbée la démultiplication de l'espace qui va de la chambre dans la tête de Torrance à celle, extérieure à cette tête mais intérieure à l'hôtel, qui capturera cette même tête, le roman, c'est celui-ci. Il sera signé Stephen King et incubera dans ma tête de lecteur.

Dans le film au contraire, c'est déjà d'un roman qu'il s'agit, comme si le script avait précipité la maturation énoncée par le livre. Que veut dire cette transformation, alors qu'on s'attendrait, compte-tenu des rapports entre théâtre et cinéma, que Kubrick garde cette forme de spécularité dont Coen, dans Barton Fink, a su tirer l'effet que l'on sait : par ce qui revient à un étonnant écrasement de la perspective, le personnage central, auteur de théâtre, est littéralement capturé par le cinéma, comme je le montrerai plus loin. Rien de tel chez Kubrick. Mais c'est que ce dernier réserve au spectateur ce que Coen fait subir au personnage. J'y reviendrai.

Autre transformation du livre au film, étrangement symétrique celle-là : le syndrome de la page blanche, chez King, devient au contraire, chez Kubrick, la répercussion folle en forme de figures géonétriques. Le texte dès lors, réduit à la visualisation sidérante de son tracé, devient surface labyrinthique et, quant à la forme de son contenu, connote le collectif, comptine ou proverbe : "Jack has been a good boy" dans la version originale et transcrit dans la version française en «un tiens vaut mieux que deux tu l'auras». L'infantilisation en anglais se transcrit en proverbialisation, mais c'est dans les deux cas une forme de «minorisation» du message.

6 Je parle de «pseudo-photogramme» parce que, bien évidemment, cette photo n'est pas un photogramme du film, encore qu'à un certain moment, sans doute, sur la pellicule, elle finisse par occuper toute la place, coïncidant alors avec le photogramme. Par rapport au film, cette photo pourrait aussi bien représenter les rushes ou les photos de plateau, c'est-à-dire des «doubles» labyrinthiques du photogramme, doubles synchrones mais que soit leur fonction (photo de plateau) soit leur «qualité» (rushes) aura contraint de rester hors de l'espace et du temps du film.

7 Il y a, en fait, deux «photogrammes» successifs, celui, en couleurs de Torrance gelé, le seul des deux qui en fait, pour cette raison, et surtout parce qu'il fait partie, fût-il plan fixe après la folie des travellings précédents, du déroulement du film, ait droit à cette appellation et celui en noir et blanc de la photo du bal. Ce dernier, la caméra, au bout d'un long et lent travelling analogue à certains de ceux qui ont parcouru l'hôtel, l'isole au milieu d'autres photos disposées de façon symétriques sur un mur, un fondu puis simplement un montage cut isolant, à l'aboutissement du travelling, en deux temps, Nicholson d'abord en plan moyen puis en plan rapproché. Puis la caméra descend pour cadrer l'inscription en bas de la photo. Le rétrécissement du champ dans la succession des plans montés de deux façons différentes succédant à un mouvement d'avancée de la caméra et donnant lieu à un mouvement vertical de la même caméra : on a là, en guise de signature, les fondements même du cinéma.

8 Voir les nombreux personnages qui, comme Torrance dans The Shining, sont des écrivains et surtout ceux, plus récents, où ils se trouvent eux-mêmes martyrisés par les monstres qu'ils ont créés : le personnage comme frère mort né, The Dark Half ou le public comme mère abusive, Misery. Il y 
aurait beaucoup à dire sur ces configurations nées sous la plume d'un écrivain dont les tirages sont les plus importants au monde, après la Bible et Marx.

9 Car cela fait aussi partie de la leçon labyrinthique.

10 Cette pluralité, qui est d'abord celle des divers meurtres, suicides et méfaits commis dans l'hôtel, n'apparaît dans le film que sous forme d'images énigmatiques : une femme jeune puis vieille et décomposée rencontrée dans une salle de bains, un homme le crâne fendu qui porte un toast, un lapin à genoux occupé à faire des choses à un homme en smoking. Bien sûr, c'est de regard qu'il s'agit : voyeurisme dans le premier et le troisième cas, exhibitionnisme dans le second. Dès lors que, contrairement au roman, aucune justification diégétique ne vient récupérer ces scènes, elles se présentent comme des «restes» d'un autre discours, semblables aux restes diurnes intégrés dans les rêves, cet autre discours étant ici le texte ainsi littéralement «caviardé». Ce qui reste énigme pour le spectateur est ironie pour le cinéaste, au même titre, bien que sous une autre forme, que la production visuelle de véritables «flots de sang» par lesquels Kubrick se moque, non point tant des romans que des films d'horreur. La mise en images de Kubrick joue volontiers du sarcasme. Voir, par exemple, le labyrinthe kitsch en quoi est organisée la chambre du cuisinier noir entre les pieds duquel la caméra, avec lui, «regarde» la télé.

11 Elle est d'ailleurs placée dans une position symétrique : le film, en effet, étant découpé en pseudo-chapitres avec titre, la séquence de la voiture d'enfant apparaît dans le III, titré «Un mois plus tard», juste après un plan fixe d'ensemble de l'hôtel, exactement comme la coccinelle des Torrance apparaissait dans le générique, juste après un plan fixe de montagne, plan bientôt animé en travelling avant.

12 Il faut cependant signaler l'exception que représente à cet égard la séquence avec le barman dans la salle de bal déserte, juste après la séquence de l'enfant jouant sur le tapis, reprise un peu plus tard (rencontre avec Grady) dans la salle de bal cette fois pleine de monde.

13 À ce propos, voir Françoise Frontisi-Ducroux, Dédale, mythologie de l'artisan en Grèce ancienne (Paris : Maspero, 1975).

14 Mais dans le texte, c'est plutôt Wendy qui occupe la place d'Icare. Qu'on en juge plutôt par ce que le texte dit de sa découverte du paysage, en arrivant à l'hôtel: "For Wendy, it was discovering truth in a cliché : her breath was taken away. For a moment she was unable to breathe at all; the view had knocked the wind from her.(...) The whole valley floor was spread out below them, the slopes that they had climbed in the laboring bug falling away with such dizzying suddenness that she knew to look down there for too long would bring on nausea and eventual vomiting. The imagination seemed to spring to full life in the clear air, beyond the rein of reason, and to look was to helplessly see one's self plunging down and down and down, sky and slopes changing places in slow cartwheels, the scream drifting from your mouth like a lazy balloon as your hair and your dress billowed out..." (pp. 63-64).

15 Il me semble que Kubrick a utilisé dans ce sens la capacité assez rare qu'a Nicholson de littéralement «changer de visage». 